\title{
Wahrnehmung \\ des Klimawandels \\ in der Metropolregion Hamburg
}

Beate M. W. Ratter

13.1 Einflussfaktoren bei der Klimawandel-

und Risikowahrnehmung - 256

13.2 Studien zur Klimawandelwahrnehmung

in der Metropolregion Hamburg - 258

13.3 Resümee: Wahrnehmung und die soziale Konstruktion des Klimawandels - 261

Literatur - 262 
„Auch wenn die Hochwasserschutzanlagen in Hamburg einem sehr hohen Schutzstandard entsprechen, erhält die Aufgabe, das Bewusstsein für die Sturmflutgefahr in der Bevölkerung wach zu halten, durch den Klimawandel noch größere Bedeutung“" (Bürgerschaft der Freien und Hansestadt Hamburg 2013). Was die Hansestadt Hamburg in ihrem Aktionsplan zur Anpassung an den Klimawandel betont, spiegelt den Kern der sozialwissenschaftlichen Klima- und Risikoforschung wider. Das Wissen um das Risikobewusstsein der Bevölkerung ist eine wichtige Größe im Risikomanagement. Wenn es in den Köpfen der Menschen keinen Platz für persönliches präventives Handeln und aktiven Schutz im Katastrophenfall gibt, wird es auch für alle weiteren Maßnahmen schwer werden, wirksam zu greifen (Scannell und Gifford 2011). Denn das Zusammenspiel des staatlichen und persönlichen Risikomanagements kann nur dann wirksam funktionieren, wenn das Risiko auch auf der persönlichen Ebene richtig eingeschätzt wird (Kates und Wilbanks 2003; Martens et al. 2009). Das Thema Risikowahrnehmung bekommt auch praktische Relevanz, wenn es darum geht, das Bewusstsein für die vorhandene Bedrohung mit geeigneter Risikokommunikation in der betroffenen Bevölkerung zu steigern.

Bewusstsein für die Bedrohung durch den Klimawandel setzt voraus, dass der Klimawandel als Bedrohung wahrgenommen wird (vgl. Hulme 2009; Kahan et al. 2012; Leiserowitz 2004, 2005), und diese Bedrohungswahrnehmung wiederum ist hinreichende, wenn auch nicht ausreichende Voraussetzung für ein Handeln zur Anpassung (z. B. Kates und Wilbanks 2003; Leiserowitz 2007). Aber warum ist das Thema Klimawandel für den Laien so schwer verständlich, und welche Faktoren beeinflussen die Wahrnehmung von Klimawandel?

Eingebettet in den wissenschaftlichen Diskurs zur Wahrnehmung des Klimawandels wird im Folgenden der aktuelle Forschungsstand zur Wahrnehmung von Klimawandel in Hamburg und Norddeutschland dokumentiert und untersucht, zu welchen Ergebnissen die vorliegenden Studien gelangt sind. Fühlt sich die Bevölkerung durch den Klimawandel bedroht? Welche Rolle spielen Erfahrungen und Einstellungen bei der Bereitschaft, etwas gegen den Klimawandel zu tun?

\subsection{Einflussfaktoren bei der Klimawandel- und Risikowahrnehmung}

Welches Risiko für die Bewohner einer Region besteht und wie proaktiv mit diesem Risiko umzugehen ist, wird von der Wahrnehmung der auftretenden Gefahren bestimmt. Das „Wahrnehmen“ eines Risikos ist ein subjektiver Einschätzungsprozess, eine mentale Konstruktion eingebettet in und bestimmt durch die Kultur einer Gesellschaft (Crona et al. 2013; Barnett und Breakwell 2001). Die individuelle subjektive Risikoeinschätzung ist intuitiv und unbewusst: „Risk perception is all about thoughts, beliefs and constructs" (Sjöberg 2000a, S. 408, 2000b). Spricht man im Alltag über Wahrnehmung, so verbirgt sich hinter dem Begriff eine Beurteilung bzw. Bewertung von Situationen oder Objekten, ohne dass hierbei auf exakte Daten oder Modelle zurückgegriffen wird. Die subjektive Wahrnehmung bzw. Risikowahrnehmung und die Logik, der sie folgt, ist vielschichtig. So werden die Einflussfaktoren eines Risikos durch eine Reihe von persönlichen Urteilen, Prinzipien und Haltungen bestimmt, die u. a. aufgrund von Erfahrung und Einschätzungen zustande kommen (Plapp und Werner 2006). Unter Wahrnehmung im kognitiven Sinn können „alle mentalen Prozesse verstanden werden, bei der eine Person über die Sinne Informationen aus der Umwelt (physisch ebenso wie kommunikativ) aufnimmt, verarbeitet und auswertet" (Renn et al. 2007, S. 77). Individuelle Risikowahrnehmungen sind nicht völlig beliebig, sondern folgen gewissen affektiven und kognitiven Mechanismen der mentalen Informationsverarbeitung, und diese finden in bestimmten gesellschaftlichen Kontexten (kulturelle Bezüge, soziale und politische Institutionen) statt (Renn 2008, S. 141-145). Sie unterliegen demnach unterschiedlichen Einflussfaktoren auf der individuellen und der gesellschaftlichen Ebene.

Die Wahrnehmung des Risikos Klimawandel ist bereits auf der affektiven und kognitiven Ebene mit einem Problem konfrontiert. Klimawandel ist eine Konstruktion, so wie „Klima“ eine statistische Größe ist. Der statistische Mittelwert Klima lässt sich vom Einzelnen nicht fühlen. Klimawandel ist nicht „erlebbar“. Wahrgenommen werden allenfalls die Auswirkungen, die man für Folgen des Klimawandels hält oder erklärt bekommt. Was der Einzelne als Klimawandel wahrnimmt, sind in vielen Fällen wetterbezogene Phänomene. Wie wirkmächtig solche Alltagsvorstellungen über Klima durch das Erleben von Wetter sein können, beschreiben u. a. Stehr und von Storch (2010, S. 105 ff) in ihrem Buch „Klima, Wetter, Mensch“ (s. auch Moser und Dilling 2011; Whitmarsh 2008, 2011). Und wie schwierig das Thema Klimawandel für den „Normalbürger“ zu verstehen ist, haben bereits Kempton (1991) und Bostrom et al. (1994) dargelegt, indem auch sie aufzeigen, dass u. a. häufig Wetter mit Klima gleichgesetzt wird oder dass die Ausdünnung der Ozonschicht mit dem Treibhauseffekt verwechselt wird. Für das Verstehen der vielschichtigen und miteinander in Beziehung stehenden Informationen über Klimawandel wird nach Sterman und Booth Sweeney (2007) im Alltäglichen auf einfache, statische mentale Modelle zurückgegriffen, um die Komplexität der Informationen zu komprimieren, oder mit bereits erlebten Phänomenen gleichgesetzt, die bereits affektiv oder kognitiv wahrnehmbar waren.

Was für den Einzelnen Klimawandel bedeutet, hängt also von den vorhandenen Informationen, der mentalen Informationsverarbeitung und den eigenen Erfahrungen ab. Unabhängig davon, welche Information zur Verfügung steht, wird die Vorstellung von Klimawandel persönlich und individuell konstruiert (Weber 2010).

Welche Faktoren darüber hinaus die Wahrnehmung von Klimawandel beeinflussen oder die Meinung zu Klimawandel bestimmen, untersuchten u. a. Capstick et al. (2015) anhand einer Metaanalyse von Studien aus den letzten Jahrzehnten. $\mathrm{Zu}$ den wichtigsten Einflussfaktoren für die unterschiedliche Wahrnehmung von Klimawandel gehören u. a. der regionale naturräumliche und soziale Kontext der Befragten (Akerlof et al. 2012), der Grad der Risikoexponiertheit gegenüber Naturereignissen, die zu Katastrophen werden können (Hartmuth 2001; Martens et al. 2009; Myers et al. 2012), kulturelle Werte und der politische Kontext (Adger et al. 2013; Devine-Wright 2013a) und nicht zuletzt auch die Art und Weise der medialen Aufbereitung 
und Verbreitung (s. Arlt et al. 2010; Capstick et al. 2015). Eine besondere Rolle nehmen Schlüsselereignisse ein, die im gesellschaftlichen Gedächtnis verankert bleiben (z. B. Hartmuth 2001) oder politische Schlüsselereignisse (z. B. das Kyoto Protokoll, s. Krosnick et al. 2000; Brechin 2003), soweit sie nicht durch periodische Wiederholung zu Ermüdungs- und Abstumpfungserscheinungen führen wie die zahlreichen, in der Öffentlichkeit meist als erfolglos eingestuften UN-Klimagipfel. Auch künstlerisch oder medial aufbereitete Beiträge tragen zur Meinungsbildung bei (etwa die Filme „The Day after Tomorrow“ von 2004 und „An Inconvenient Truth“ von 2007).

Zur Klimawandelwahrnehmung im regionalen, naturräumlichen und sozialen Kontext betonen Adger et al. (2013) die Bedeutung der soziokulturellen Rahmung der Wahrnehmung des Klimawandels und verweisen darauf, dass diese gesellschaftliche Dimension nicht nur die Wahrnehmung beeinflusst, sondern dass sie auch den gesellschaftlichen Umgang mit dem Klimawandel im Alltagshandeln des Einzelnen bestimmt. Gerade in den vergangenen Jahren wurde in zahlreichen Studien festgestellt, dass Klimawandelwahrnehmung ortsabhängig und kulturell gerahmt ist (Capstick et al. 2015; Brechin und Bhandari 2011; Renn und Rohrmann 2000). Studien zur Klimawandelwahrnehmung in den peruanischen Anden (Paerregaard 2013), in Norwegen (Ryghaug et al. 2011), in Sibirien (Lavrillier 2013), in Großbritannien (Capstick 2012), bei Hispanics in den USA (Davenport 2015) oder bei Migranten in Hamburg (de Guttry et al. 2016) verweisen auf die orts- und kulturabhängige Verankerung von Klimawandel in täglichen Praktiken und Ritualen sowie deren Wandel im Laufe der Zeit. Dabei spielen u. a. ortsbezogene Wetterphänomene und Extremereignisse eine entscheidende Rolle, auch unterschiedliche kulturelle Einstellungen $\mathrm{zu}$ Umwelt und Natur zeigen hier ihre Wirkung (Adger et al. 2013; Devine-Wright 2013a, 2013b, 2015b; Dunlap 1998; Upham et al. 2009; Wolf und Moser 2011). Diese kulturellen Einstellungen zu Umwelt und Natur finden sich in sog. gesellschaftlichen „Archiven“ historischen Wissens wieder, die nicht unabhängig von spezifischen Räumen zu sehen sind. Nach Meusburger (2006) bilden sich in bestimmten Räumen durch historische Sedimentierungen von Wissen bestimmte Strukturen, die für die Wahrnehmung und das Handeln der Menschen orientierend wirken. Meusburger (2006, S. 280) weist darauf hin, dass das spezifische historische Wissen eines (Kultur-)Raumes eine entscheidende Bedeutung für die Entstehung persistierender Pfadabhängigkeiten hat. Die Aufnahme und Verbreitung neuen Wissens im spezifischen räumlichen Kontext kann dadurch beeinflusst werden (vgl. Christmann et al. 2012, S. 31). Dementsprechend belegen die genannten Studien, dass Klimawandelwahrnehmung auf der regionalen Ebene entscheidend ist, dass Klimawandelwahrnehmung ortsgebunden und gesellschaftlich gerahmt ist. Diese regionalen und lokalen Wahrnehmungsmuster gilt es offenzulegen, weil sie für die Diskussion und Planung regional eingebetteter Anpassungsstrategien wichtig sind (Arbuckle et al. 2013; Brügger et al. 2015; Chess und Johnson 2007; Döring und Ratter 2017; Taylor et al. 2014a, 2014b; Weber und Stern 2011).

Klimawandelwahrnehmung findet darüber hinaus in einem bestimmten politischen Kontext statt. So spielt in demokratischen Gesellschaften Wahrnehmung nicht nur für das eigene
Handeln eine entscheidende Rolle, sondern es bestimmt auch die politische Willensbildung und politische Entscheidungsprozesse der Wähler, die nicht zuletzt auf der Basis ihrer Einstellungen zur Erteilung oder eben Nichterteilung eines Mandats zur aktiven Gestaltung des Umgangs mit dem Klimawandel beitragen (Dietz und Stern 2008; Lorenzoni et al. 2007; Shwom et al. 2010). Interessant ist dabei, dass die Bedeutung von naturwissenschaftlichem Wissen für die Wahrnehmung von Klimawandel und für die eigene wertende Positionierung dazu als nur randständig wichtig betrachtet werden kann und vielmehr von den Werten und der Weltanschauung des Einzelnen abhängt (Devine-Wright 2013a, 2013b, 2015a, 2015b). So verläuft z. B. die Anerkennung von Klimawandel als anthropogenes Phänomen in den USA entlang parteipolitischer Trennlinien (Brewer 2012; Guber 2013; Krosnick et al. 2000). Meinungsbilder entwickeln sich im Kontext gesellschaftlicher Prozesse (Kahan et al. 2012; O’Neill und Hulme 2009; Raaijmakers et al. 2008). Kahan et al. (2012) konnten in ihrer Studie zeigen, dass die Einschätzung von Klimawandel viel eher von Interessenkonflikten bestimmt wird und die Haltung zum Klimawandel sich entlang der Gruppenbildungsprozesse von Gleichgesinnten ergibt.

Eine entscheidende Rolle spielen Schlüsselereignisse und die Risikoexponiertheit gegenüber Naturereignissen. Nach Hartmuth (2001, S. 243) beeinflussen extreme Wetterereignisse mit lokalen Auswirkungen die soziale Repräsentation des anthropogenen Klimawandels, und ein wichtiger Faktor ist die Erfahrung mit Extremereignissen in der Vergangenheit (Whitmarsh 2008; Grant et al. 2015). Dabei kommt auch der Art und Weise der medialen Aufbereitung, Verbreitung und Verankerung in der Berichterstattung eine zentrale Rolle zu (Trümper und Neverla 2013; Arlt et al. 2010).

Neben der räumlichen und soziokulturellen Unterschiedlichkeit von Klimawandelwahrnehmung unterliegt die Wahrnehmung auch einer zeitlichen Veränderung. Capstick et al. (2015) unterscheiden vier Phasen der Klimawandelwahrnehmung, die sie seit den Anfängen der breiteren Untersuchung in den 1980er-Jahren identifiziert haben. Nach einer frühen Phase der wachsenden Sensibilisierung (1980 bis frühe 1990er-Jahre) folgte eine Phase des Anstiegs und der Betroffenheitsschwankungen (Mitte 1990 bis Mitte 2000); danach zeigte sich eine Phase von wachsendem Skeptizismus und Polarisierung (bis späte 2000erJahre), die anschließend in eine neue Phase der öffentlichen Wahrnehmung (seit Ende der 2000er- bis zu den frühen 2010erJahren) mündete. Sicherlich sind diese Phasen nicht trennscharf voneinander abzugrenzen und bedürfen darüber hinaus der empirischen Untermauerung in verschiedenen soziokulturellen Kontexten. Allerdings erweist es sich dabei als problematisch, dass die meisten Wahrnehmungsuntersuchungen nur Momentaufnahmen darstellen und nur sehr wenige konsistente Langzeitstudien vorliegen. So können sich vermeintlich ausgemachte rückläufige Trends bei genauerer Betrachtung auch als bloße Schwankung erweisen (Ratter et al. 2012).

Insgesamt bleibt festzuhalten, dass Wahrnehmungsforschung in einem konkreten gesellschaftlichen Kontext steht, der für die Analyse der erhobenen Konstruktionen von Klimawandel und Einschätzung von Klimawandelfolgen mit zu berücksichtigen ist. Bereits die frühen Studien von Bostrom et al. (1994) zum 
(Nicht-)Wissen oder zum Verständnis von US-Bürgern über den Klimawandel oder Studien von Weber (2008) zu den Alltagsbildern des Klimawandels und zum Klimabewusstsein in Deutschland verdeutlichen die Notwendigkeit der Kontextualisierung und der gesellschaftlichen Analyse von Klimabild und Klimawahrnehmung in den Köpfen der Menschen (s. auch Lorenzoni und Pidgeon 2006).

\subsection{Studien zur Klimawandelwahrnehmung in der Metropolregion Hamburg}

Auch wenn insgesamt eine fast unüberschaubare Vielzahl an Publikationen zum Thema Wahrnehmung des Klimawandels existiert ( Abschn. 13.1), ist das Feld der öffentlich zugänglichen Studien und wissenschaftlichen Beiträge für Hamburg und den norddeutschen Raum überschaubar. Für die vergangene Dekade konnten insgesamt 10 Studien und Forschungsprojekte identifiziert werden, die sich mit Fragen der Wahrnehmung und Risikoeinschätzung von Klimawandel in der Metropolregion Hamburg und im norddeutschen Küstenraum befassen; speziell für Hamburg konnten nur zwei Wahrnehmungsstudien identifiziert werden. Einige der Studien beziehen sich dabei vornehmlich auf die Wahrnehmung von Sturmfluten sowie die Risiken des Küstenschutzes und stehen nur mittelbar im Kontext der Klimawandeldiskussion. Diese einzelnen Studien werden im Folgenden vorgestellt - unter dem besonderen Augenmerk der oben beschriebenen Einflussfaktoren auf die Klimawandelwahrnehmung.

Am Leibniz-Institut für Raumbezogene Sozialforschung e. V. (IRS) in Erkner entstanden im Zusammenhang mit dem Potsdamer Forschungs- und Technologieverbund zu Naturgefahren, Klimawandel und Nachhaltigkeit (PROGRESS) im Teilprojekt „D3.1 - Gesellschaftliche Verarbeitungen von Klimarisiken“ mehrere Untersuchungen zum Klimawandel in deutschen Küstenstädten und Gemeinden, die sich u. a. mit der sozialen Konstruktion von Klimawandel sowie mit Handlungsempfehlungen beschäftigten (Balgar und Mahlkow 2013; Christmann et al. 2012; Heimann und Christmann 2013; Heimann und Mahlkow 2012). Dabei liegt insbesondere eine vergleichende Analyse von lokaler Konstruktion zu Vulnerabilität und Resilienz in Lübeck und Rostock vor (Christmann et al. 2014). In dieser Studie konnte nachgewiesen werden, dass Wahrnehmungsunterschiede von Klimarisiken größer sind als angenommen und dass lokale Vorstellungen über den Klimawandel in spezifische städtische Traditionslinien bzw. Pfade eingebettet sind und sich infolgedessen unterscheiden können. Die Analysen der Mediendiskurse weisen darauf hin, dass - vermittelt über internationale und nationale Diskurse - mögliche Bedrohungen durch den Klimawandel zwar wahrgenommen werden, die Art und Weise der Wahrnehmung sich jedoch unterscheidet, je nachdem, in welche kulturellen Traditionslinien und lokalen Wissensbezüge sie eingeordnet werden (Christmann et al. 2014, S. 32).

Im Projekt COMRISK wurde u. a. die Beziehung zwischen Sturmflutrisiken und Sturmflutmanagement untersucht (Kaiser et al. 2004; Birkholz et al. 2014) sowie raum-zeitliche Muster von Flut-Anpassungsmaßnahmen auf Haushaltsebene identifiziert (Koerth et al. 2014). Im Rahmen des Teilprojekts 3 („Public perception of coastal flood defence and participation in coastal flood defence planning"; vgl. Kaiser et al. 2004) wurde mithilfe einer standardisierten Befragung entlang der Nordseeküste in Dänemark, Deutschland, Niederlande, Belgien und dem Vereinigten Königreich die Risikowahrnehmung von Sturmfluten von Bürgern sowie deren Partizipation an Küstenschutzmaßnahmen untersucht. Ziel des Projektes war es, vorhandene Informationsmethoden zu verbessern, um die lokale Bevölkerung für Sturmflutrisiken und Küstenschutzmaßnahmen nachhaltig zu sensibilisieren. Die Untersuchung der Wahrnehmung von Klimawandel als solches spielte hierbei allerdings keine Rolle.

Im KRIM-Projekt wurden die Interpretationen des globalen Klimawandels durch die Öffentlichkeit und ihre Konsequenzen für die Risikowahrnehmung und die Implementierung eines vorbeugenden Küstenschutzes untersucht (Peters und Heinrichs 2004). Es ging dabei um die Analyse der bestehenden Bedeutungskonstruktionen von Klimawandel und Sturmflutrisiken (Peters und Heinrichs 2005) sowie um Alltagsbilder und Bewusstsein des Klimawandels (Weber 2008; Heinrichs und Grunenberg 2009). In einer dreiteiligen Studie aus Medienanalyse, Interaktions- und Rezeptionsstudie mit Experten, Journalisten und Bürgern aus Bremen, Wilhelmshaven und dem Wangerland wurden Risikovorstellungen zu den Themen Klimawandel und Küstenschutz untersucht. Peters und Heinrichs (2005) konnten zeigen, dass die Vorstellungen von Klimawandel in weiten Teilen durch den klimawissenschaftlichen Diskurs beeinflusst sind, während Vorstellungen über Sturmfluten hauptsächlich durch lokal- bzw. regionalpolitisch-administrative Institutionendiskurse geprägt werden. Insbesondere die Bevölkerung zeigte hohes Vertrauen in die Küstenschutzmaßnahmen und fühlte sich vor diesem Hintergrund auch vom Klimawandel wenig bedroht (Peters und Heinrichs 2005, S. 191).

Im EU-Interreg-Projekt SAFECOAST - Sustainable Coastal Risk Management - Subproject: „The Informed Society“ ging es um die Frage der zukünftigen Küstenschutzstrategie für 2050 und die Konsequenzen des angenommenen Klimawandels für die Raumentwicklung und die öffentliche Sicherheit gegenüber Sturmflutrisiken. Aufbauend auf empirischen Erhebungen und Analysen des Sturmflut-Risikobewusstseins der Bevölkerung in den Nordsee-Anrainerstaaten wurde Informationsmaterial für die Küstenbevölkerung Schleswig-Holsteins über Sturmflutrisiken und individuelle Verhaltensmöglichkeiten erarbeitet (Heinrichs et al. 2007). Auch in diesem Projekt spielte der Klimawandel nur indirekt und die Klimawandelwahrnehmung gar keine Rolle.

Im Rahmen des BMBF-Projektes „Integriertes Hochwasserrisikomanagement in einer individualisierten Gesellschaft" (INNIG) verglichen Heinrichs und Grunenberg (2007) in einer empirischen Studie zu Klimawandel und Gesellschaft unterschiedliche Perspektiven der Adaptationskommunikation. Dabei untersuchten sie einerseits die institutionelle Risikokommunikation und die (lokale) Informationsumwelt, in der die Bürger leben; u. a. führten sie eine repräsentative Umfrage in Bremen und Hamburg mit Telefoninterviews unter je 400 Bürgern durch. In diesem Projektteil ging es um die Einschätzung der Sturmflutrisiken und des Meeresspiegelanstiegs aus Sicht der Bevölkerung. Die Studie kommt zu dem Ergebnis, dass durchaus Gemeinsamkeiten, aber auch Unterschiede nachzuweisen sind und dass die 
Bevölkerung in Bremen trotz objektiv höherer Gefährdung ein geringeres Risikobewusstsein aufweist (vgl. Martens et al. 2009). Eine Erklärung dafür könnte nach Heinrichs und Grunenberg (2009) in der unterschiedlich im kollektiven Gedächtnis verankerten Erinnerung an die Sturmflut in Hamburg 1962 bieten (vgl. Kap. 12).

Hartmuth (2001) beschäftigte sich in einer ausgewählten Fallstudie mit den Einstellungen zum Klimawandel. Im Rahmen von zwei aufeinanderfolgenden Interviewreihen untersuchte er, wie das Thema Klimawandel im Vergleich zu anderen lokalen Themen auf Sylt repräsentiert wird. Es wurden insgesamt 70 Schlüsselpersonen aus dem Natur-, Umwelt- und Küstenschutz sowie unterschiedliche Akteure aus Wirtschaft und Gesellschaft befragt. Zunächst ging es um die Aufdeckung des allgemeinen Kontextes der wirtschaftlichen, gesellschaftlichen und ökologischen Entwicklungen auf Sylt. Daran schloss sich die Analyse der Interpretation von Klimawandel und der damit in Verbindung stehenden Fragen an. Es zeigte sich, dass das Thema Klimawandel für die befragten Schlüsselpersonen nur eine untergeordnete Rolle spielte (Hartmuth 2001, S. 158 ff). Relevante Unterschiede zwischen den sozialen Gruppen konnten dabei nicht nachgewiesen werden. Im Hinblick auf mögliche Auswirkungen des Klimawandels wurden hauptsächlich Wetteränderungen (93\%), der Anstieg des Meeresspiegels (87\%) und Überschwemmungen/ Landverlust (86\%) angegeben. Sturmfluten folgten mit $59 \%$ ebenfalls als relativ häufig genannte Auswirkung des Klimawandels (Hartmuth 2001, S. 178), die insbesondere von der Gruppe der Küstenschützer genannt wurde.

In einer vom Helmholtz-Zentrum Geesthacht durchgeführten umfassenden persönlichen Straßenumfrage wurden über 800 Einwohner entlang der deutschen Nordseeküste befragt (Ratter et al. 2009). Ziel der Studie war es, ein Bild der gesellschaftlichen Sicht in der Nordseeregion zu gewinnen und die Beziehung der Küstenbewohner zu ihrer natürlichen und sozialen Umwelt, insbesondere die Wahrnehmung der Bevölkerung zu den Themen Heimat und Region, Natur und Umwelt, Gefahren und Maßnahmenbedarf sowie zukünftige Entwicklung aufzudecken. Die Studie hat gezeigt, dass die Nordsee für ihre Anwohner einerseits einen Ort der Ressourcen, andererseits aber auch einen Ort der Gefahren darstellt. Sturmfluten und Deichbaumaßnahmen sind die historischen Ereignisse, die den an der Küste lebenden Menschen geprägt haben. Gleichermaßen zeigte sich das Bewusstsein, dass der Mensch die Region maßgeblich gestaltet hat und gleichzeitig Sturmfluten in der Wahrnehmung der Küstenbewohner auch heute noch die bedeutendste Gefahr darstellen, die bedingt durch den Klimawandel in Zukunft eine noch größere Rolle spielen wird. Bei der Wahrnehmung der Gefahren gibt es kaum regionale Unterschiede. Die Gefahren werden von den Menschen in der Küstenzone Niedersachsens und Schleswig-Holsteins nur geringfügig anders gewertet. Immerhin die Hälfte der Befragten fühlt sich von diesen Gefahren persönlich betroffen. Die Menschen betonen die potenziell weit reichende Dimension der Schäden für Anwohner durch Sturmflutereignisse mit Äußerungen wie: „Jeder ist betroffen, der hier lebt - die Lebensgrundlagen sind dann weg“ oder „Dann hätte ich keine Heimat mehr" (Ratter und Sobiech 2011). Den höchsten Maßnahmen- und Schutzbedarf sehen die Befragten im Küsten- und
Inselschutz. Allerdings zeigt sich gleichzeitig, dass die Befragten mit den aktuell getroffenen Maßnahmen sehr zufrieden sind. Das hohe Vertrauen in die verantwortlichen Institutionen ist gepaart mit der Einsicht, dass es für die Befragten schwer erscheint, persönlich Schutzstrategien zu entwickeln. Für viele gibt es keine Kontroll- und Einflussmöglichkeiten auf unmittelbare Gefahren wie den Klimawandel.

Im Projekt A-Küst (Veränderliches Küstenklima - Evaluierung von Anpassungsstrategien im Küstenschutz), ein Teilprojekt in KLIFF (Klimafolgenforschung in Niedersachsen), wurden an der deutschen Nordseeküste in der Ems-Dollart-Region die regionale Wahrnehmung des Klimawandels und seine Bedeutung für Klimawandelpassungsmaßnahmen untersucht (Schmidt et al. 2014). Im Zentrum standen in diesem Projekt die Verständigung und die Zusammenarbeit unterschiedlicher Akteure in Bezug auf Klimaanpassungsstrategien im Küstenschutz. Im Rahmen des Projektes wurden u. a. mögliche Szenarien für unterschiedliche Küstenschutzstrategien vorgestellt und bewertet. Begleitend $\mathrm{zu}$ diesem Diskussionsprozess wurden im Rahmen zweier halbstandardisierter Befragungen neben der Wahrnehmung des Klimawandels und der Akzeptanz alternativer Küstenschutzoptionen durch die Bevölkerung auch die Themenfelder Verbundenheit zur Region, langfristige Perspektiven unter Klimawandelbedingungen sowie Nutzungsänderungen und damit verbundene potenzielle Konflikte untersucht. Die beiden Bevölkerungsbefragungen haben gezeigt, dass das wissenschaftliche Wissen über Ursache und Wirkung des Klimawandels in der Bevölkerung nur unvollständig bis fehlerhaft vorhanden ist. Diese Unsicherheit ist allerdings gleichzeitig mit einem ausgeprägten Vertrauen in die Küstenschutzinstitutionen verknüpft (Behörden, Deichverbänden, Wissenschaft), das sich vor allem auf die positiven Erfahrungen der letzten Jahrzehnte stützt. Das festgestellte gegenwärtig geringe Bedrohungsgefühl durch den Klimawandel kann mitunter auf dieses starke Vertrauen zurückgeführt werden (Schmidt et al. 2014).

Das Verbundprojekt KLIMZUG Nord beschäftigte sich vornehmlich mit raumplanerischen Aspekten und den Herausforderungen der Anpassungsstrategien an den Klimawandel. Das Projekt REKLIMAR, in dem eine Studie zu Perspektiven für klimaangepasste Innovationsprozesse in der Metropolregion Bremen-Oldenburg im Nordwesten durchgeführt wurde, befasste sich mit der Wahrnehmung des Klimawandels im Alltag und seinen Folgen für Konsumverhalten und Vulnerabilität in der Nordwest-Region (Weller et al. 2010; Krapf und Weller 2013). Anhand einer quantitativen Erhebung wurden repräsentative Aussagen darüber gewonnen, welche Einstellungen die Bewohner der Metropolregion Bremen-Oldenburg gegenüber Klimawandel, Klimaschutz und Klimaanpassung haben und welches Meinungsbild gegenüber erneuerbaren Energien und damit verbundenen Entwicklungen besteht (Weller et al. 2010). REKLIMAR zielte zudem darauf, den Einfluss sozioökonomischer und raumbezogener Faktoren auf die Wahrnehmung des Klimawandels und seiner Folgen in der Region zu bestimmen. Die Ergebnisse zeigen, dass das Problem des Klimawandels im Alltag der Bürger der Nordwest-Region präsent ist. Dies zeigt sich $u$. a. darin, dass subjektiv wahrgenommene Umweltveränderungen mit dem Klimawandel assoziiert werden, bei klimaschädlichem Verhalten ein schlechtes 


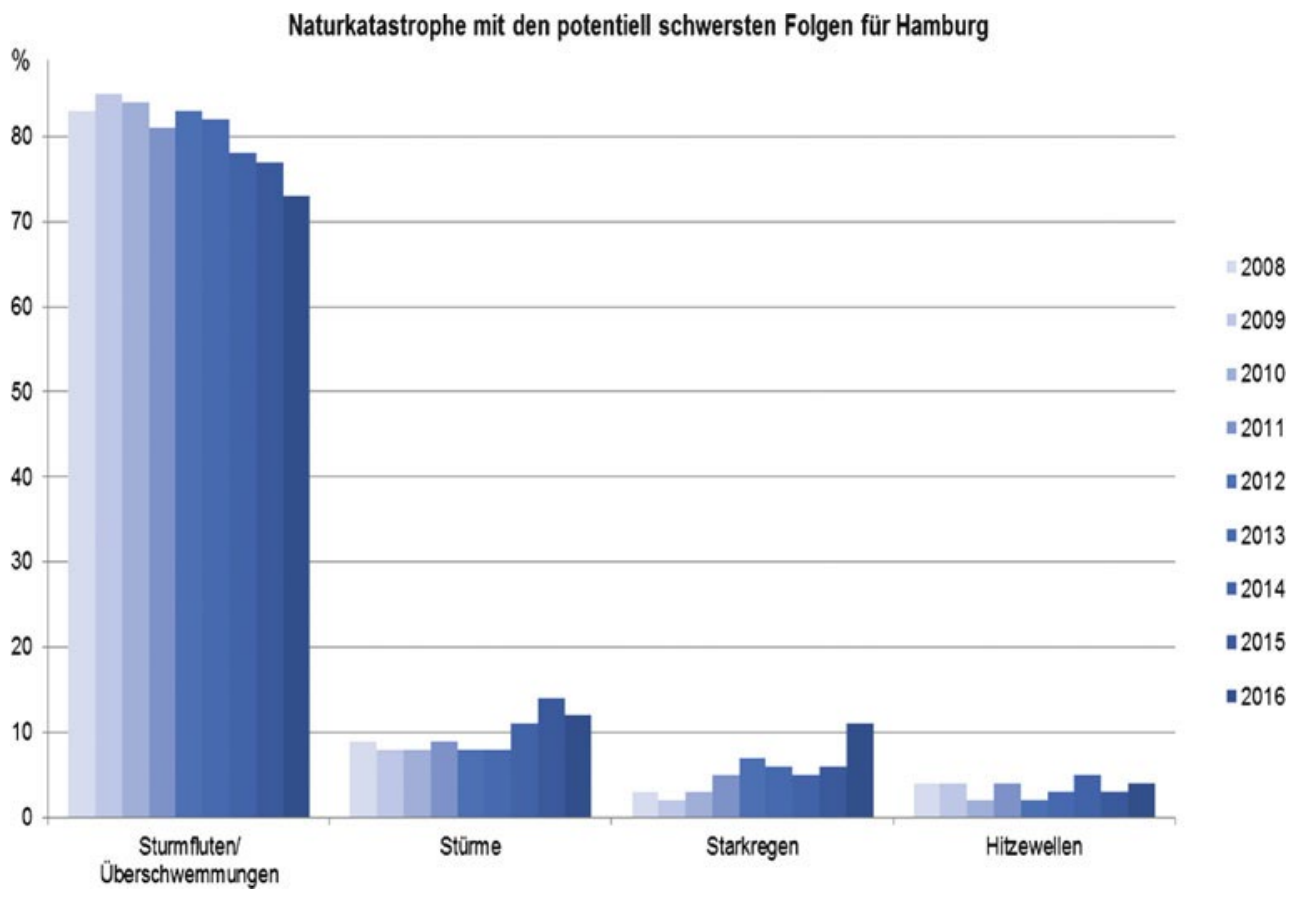

- Abb. 13.1 Einschätzung der Befragten: Naturkatastrophe mit den potenziell schwersten Folgen für Hamburg (2008-2016)
Gewissen besteht oder auch über mögliche Anpassungsmaßnahmen an die Folgen des Klimawandels nachgedacht wird. Allerdings besteht, obgleich der Klimawandel bei den Bürgern präsent ist, in mehrfacher Hinsicht Unsicherheit, und es zeigte sich laut Krapf und Weller (2013), dass die festgestellten gruppenspezifischen Unterschiede die Notwendigkeit zielgruppenspezifischer Strategien klimawandelbezogener Information und Kommunikation unterstreichen (Krapf und Weller 2013, S. 50).

In einer umfassenden Studie des Bundesexzellenzclusters CliSAP an der Universität Hamburg wurde mit einer deutschlandweiten Umfrage systematisch der Zusammenhang zwischen vorhandener Klimawandelskepsis, Energiepräferenz und politischer Partizipation untersucht (Engels et al. 2013). Die Autoren gehen davon aus, dass Klimawandelwahrnehmung auch die Basis für Entscheidungen für oder gegen $\mathrm{CO}_{2}$-reduzierende Maßnahmen bildet, und dies gilt sowohl in der Politik als auch beim Einzelnen (Whitmarsh 2011). Im Fokus der Untersuchung stand die Beziehung zwischen Klimaskepsis und Unterstützung von Mitigations- (z. B. Energiepräferenzen, Sparmaßnahmen) oder Anpassungsmaßnahmen von privaten Haushalten und Bürgerbeteiligungsaktionen, bei denen die Wahrnehmung eine zentrale Rolle spielt. Deutschland wird hier, wegen seiner weitreichend diskutierten „Energiewende“, als „interessantes Testfeld“ bezeichnet. Die Ergebnisse der Studie verweisen darauf, dass es in Deutschland vergleichsweise weniger Klimaskeptiker gibt als z. B. in den USA, solche Zweifler allerdings häufig negativ mit politischer Beteiligung und mit einer Unterstützung erneuerbarer Energien korrelieren.

Neben den umfassenderen Studien zur Klimawandelwahrnehmung aus dem norddeutschen Kontext gibt es zwei Studien, die sich spezifisch mit Hamburg und den Hamburger Bürgern befassen. Das Forschungslabor der Fakultät Wirtschafts- und Sozialwissenschaften der Universität Hamburg führt seit 2012 unter der Leitung von Kai-Uwe Schnapp jährlich Umfragen unter Hamburger Bürgern durch. Fragen zum Klimawandel wurden allerdings nur in der ersten Runde der sozialwissenschaftlichen Umfragestudie behandelt. Die erste Befragung fand zwischen dem 16. April und dem 3. August 2012 statt. Die klimabezogenen Themen begannen mit der Frage, inwiefern der Klimawandel ein ernsthaftes Problem darstellt. $69 \%$ der Befragten stimmten der Aussage „voll und ganz“ zu, weitere $18 \%$ stimmten dieser Aussage „eher zu“ als nicht zu. 55 \% befürchteten eine Zunahme von Sturmfluten, weitere $27 \%$ sahen eine wachsende Bedrohung durch Überschwemmungen. Schaut man auf die Wählerschaft der einzelnen Parteien, werden Unterschiede deutlich. So waren 82 \% der Wähler der Linkspartei voll und ganz der Meinung, dass der Klimawandel ein ernsthaftes Problem ist; bei SPD, Grünen und Piraten waren es je $75 \%$. In der Wählerschaft der FDP lag die volle Zustimmung zu dieser Aussage bei $66 \%$ und bei den Wählern der CDU bei 55 \%. 94 \% der Befragten stimmten der Aussage, dass der Mensch der Umwelt ernsthaften Schaden zufügt, „voll und ganz“ oder „eher zu“ (Bock und Schnapp 2012, S. 18).

Seit 2008 wird jährlich das Risikobewusstsein der Hamburger Bürger für den Klimawandel in einer Umfrage vom Institut für Küstenforschung des Helmholtz-Zentrums Geesthacht erhoben. In Zusammenarbeit mit dem Meinungsforschungsinstitut Forsa werden in einer Telefonumfrage ca. 500 Bürger in Hamburg über ihre Einschätzung zum Einfluss des Klimawandels auf ihr Leben sowie nach der Wahrscheinlichkeit des Eintretens von Naturkatastrophen befragt. Die Frage „Stellt der Klimawandel Ihrer Meinung nach eine sehr große, eine große, eine weniger große oder überhaupt keine Bedrohung für Hamburg dar?" wurde dabei zwischen 2008 und 2016 überwiegend mit "groß“ bis „sehr groß“ eingestuft. Nach einem Rückgang zwischen 2010 und 2012, bei dem über die Hälfte der befragten Hamburger den Klimawandel nicht als "große“ oder „sehr große“ Bedrohung wahrgenommen hatte, sahen in den vergangenen drei Jahren zunehmend mehr Befragte den Klimawandel als Bedrohung für Hamburg („sehr groß“ 15 \%, „groß“ 41 \%). Seit 2011 emp- 
- Abb. 13.2 Wahrnehmung der Bedrohung durch Klimawandel in Hamburg und den USA (20062015). (Vgl. auch Ratter et al. 2012)
Besorgnis bzgl. Klimawandel in Hamburg und den USA

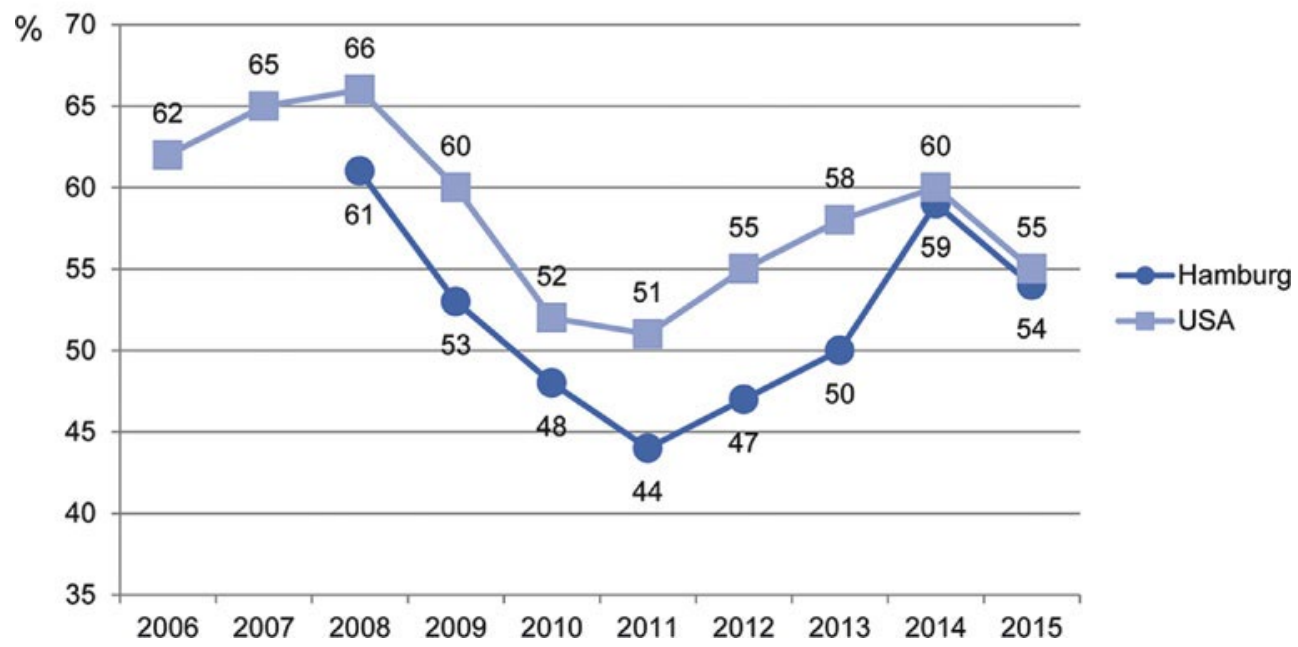

fand die deutliche Mehrheit der Befragten Hamburger spürbare Auswirkungen des Klimawandels bereits heute und nicht erst in Zukunft. Von den 39 \% (2016) der Befragten, die die Folgen des Klimawandels bereits heute wahrnehmen, sind $44 \%$ Frauen und $32 \%$ Männer. Der Anteil derjenigen, die schon heute Klimawandelfolgen bemerken, steigt mit dem Bildungsstand. Der prozentuale Anteil derer, die erst in über 30 Jahren mit Klimafolgen rechnen, ist dabei wie in den Vorjahren unter den 14- bis 29-Jährigen im Vergleich zu den anderen Altersgruppen besonders hoch (16 vs. 6-8\%).

Während in den Jahren 2008 bis 2012 Sturmfluten von der deutlichen Mehrheit der Befragten als Naturkatastrophe mit den potenziell schwersten Folgen für Hamburg wahrgenommen wurde $^{1}$, zeichnet sich seit 2013 ein neuer Trend ab: zwar nehmen 2016 immer noch 73 \% der Teilnehmer Sturmfluten als gravierendste Bedrohung wahr, dies sind allerdings $10 \%$ weniger als im ersten Umfragejahr 2008. Stattdessen stieg die Wahrnehmung von Stürmen und Starkregen als Ereignisse mit schweren Folgen für Hamburg in den letzten Jahren kontinuierlich an. Im Vergleich zu $9 \%$ (Stürme) bzw. 3 \% (Starkregen) in 2008 nehmen im Jahr 201612 bzw. 11 \% der Befragten diese Ereignisse als bedrohlich für die Stadt wahr. Vor allem Starkregenereignisse sind in der Wahrnehmung der Bürger stark angestiegen (6 auf $11 \%)$ (vgl. - Abb. 13.1). Persönlich betroffen von den Naturkatastrophen im Rahmen des Klimawandels fühlte sich ähnlich wie im Vorjahr die knappe Mehrheit der Teilnehmer (ja: 52 \%, nein: $47 \%$ ). Ein besonders großer Anteil der 14- bis 29-Jährigen beantwortet die Frage nach persönlicher Betroffenheit mit „ja“ (60\%, nein: $37 \%$ ).

Die Langzeitstudie zeigt, dass die Entwicklung der Klimawandelwahrnehmung unter Hamburger Bürgern Schwankungen

1 Die Frage nach der Naturkatastrophe, die für Hamburg die schwersten Folgen bringen wird, wurde als geschlossene Frage mit vorgegebenen Antwortkategorien gestellt: „Zu den negativen Folgen des Klimawandels können unter anderem auch Naturkatastrophen gehören. Welche der folgenden Naturkatastrophen hätte Ihrer Meinung nach die schwersten Folgen für Hamburg (Einfachnennung): Stürme, Starkregen, Hitzewellen, Sturmfluten und Überschwemmungen?" unterliegt. Nach einem Hochpunkt in 2008 und einem Tiefpunkt in 2011 scheint der Spitze von 2014 bereits wieder vorüber zu sein. Ähnliche Tendenzen zeigt der Vergleich mit US-amerikanischen Befragungsergebnissen von Gallup ${ }^{2}$, wo nach dem Anstieg von 2011 bis 2014 der Klimawandel in 2015 wieder als weniger bedrohlich eingeschätzt wird. In beiden Befragungsräumen sank der Wert 2015 um fünf Prozentpunkte. Der Hype nach der Veröffentlichung des jüngsten IPCC-Weltklimaberichtes im Jahr 2014 ist schon wieder vorbei, und andere Probleme als der Klimawandel rücken in der Wahrnehmung der Bevölkerung in den Vordergrund (Ratter et al. 2012; - Abb. 13.2).

\subsection{Resümee: Wahrnehmung und die soziale Konstruktion des Klimawandels}

Die vorliegenden Studien zur Wahrnehmung von Klimawandel in Norddeutschland und Hamburg ergeben nicht nur wegen ihrer unterschiedlichen Ziele, eingesetzten Methoden und unterschiedlichen Untersuchungsräume ein mosaikartiges Bild der Klimawandelwahrnehmung in der Bevölkerung. Sie bestätigen jedoch die in internationalen Studien herausgestellten Trends zur Klimawandelwahrnehmung und deren Einflussfaktoren.

Das Problem des Klimawandels ist im Alltag der Bürger durchaus präsent; das gilt für alle Studien gleichermaßen. Für Hamburg zeigten beide Umfragen, dass der Klimawandel für die Befragten ein „ernsthaftes Problem“ darstellt. Allerdings lässt sich einschränkend feststellen, dass Klimawandel nicht das wichtigste Problem für die befragten Bürger darstellt. Nur wenige Studien betrachten diese Entwicklung über einen längeren Zeitraum hinweg. Diejenigen, die dies tun, belegen, dass sich die Besorgnis über die Risiken durch den Klimawandel bislang auf einem gleich bleibenden, wenn auch schwankenden Niveau hält (Ratter et al. 2012). Anders als in US-amerikanischen Studien lässt sich für den deutschen Kontext keine eindeutige Verbindung zwischen par-

2 Quelle: http://www.gallup.com/poll/182150/views-climate-change-stableextreme-winter.aspx 
teipolitischer Präferenz und Einstellung zum Klimawandel feststellen.

Der Klimawandel wird vor allem mit subjektiv wahrgenommenen Umwelt- und Wetterveränderungen assoziiert. Mögliche Auswirkungen des Klimawandels werden hauptsächlich mit Wetteränderungen und erst in zweiter Linie mit dem Anstieg des Meeresspiegels oder anderen Extremereignissen wie Überschwemmungen/Landverlusten gleichgesetzt. Sturmfluten spielen hierbei eine besondere Rolle, nicht nur weil sie als kulturelle Traditionslinien mit lokalen Wissensbezügen eingeordnet werden können (vgl. Christmann et al. 2014, S. 32), sondern weil sie auch einen wichtigen Faktor als Erfahrung mit Extremereignissen in der Vergangenheit darstellen (vgl. Whitmarsh 2008; Christmann et al. 2012; Grant et al. 2015).

In den Vergleichsstudien zwischen Lübeck und Rostock sowie Hamburg und Bremen zeigte sich, dass Wahrnehmungsunterschiede von Klimarisiken größer sind als vielleicht angenommen und dass diese Unterschiede bei den lokalen Vorstellungen über den Klimawandel in spezifische städtische Traditionslinien bzw. Pfade eingebettet sind. Für Hamburg ist die Sturmflut von 1962 bis heute prägend. Die Sturmflut in Hamburg 1962 hat sich im Laufe von Jahrzehnten als Erinnerung an „Naturkatastrophen" ins kollektive Gedächtnis eingebrannt, und zugleich wird diese Erinnerung überformt und mit Themen des Klimawandels verbunden (Trümper und Neverla 2013; Kruse 2015, Kruse und Siedschlag 2012; Ratter und Kruse 2010). Der Klimawandel brachte einen neuen Begründungszusammenhang für das ständig wiederkehrende Phänomen Sturmflut in Hamburg und hat damit die Funktion eines Erinnerungsankers. Dieser Zusammenhang zwischen Sturmfluten und Klimabewusstsein wird in den regelmäßig stattfindenden Umfragen in der Hamburger Bevölkerung deutlich, die zeigen, dass die Einschätzungen der Gefährdung Hamburgs durch den Klimawandel im Anschluss an Sturmfluten etwas zunehmen, jedoch mit zeitlicher Distanz zu Sturmfluten wieder zurückgehen.

Insgesamt ergeben sich unterschiedliche individuelle Einstellungen und Bedeutungszuschreibungen zum Klimawandel, und diese sind dynamisch und verändern sich mit neuen Erfahrungen und neuen Informationen. Aber Informationen allein reichen nicht aus, um ein Engagement zu stimulieren. Information ist nicht mit Wissen gleichzusetzen und führt nicht zwangsläufig zum Handeln oder zur Umsetzung von Anpassungs- und Vermeidungsstrategien bzgl. des Klimawandels (Barnett und Breakwell 2001; Chess und Johnson 2007). Die Anpassung an den Klimawandel wird als direkte vorsorgende Schadensbegrenzung/-vermeidung verstanden. Die Akteure adressieren konkrete und erwartete Auswirkungen durch den Klimawandel (z. B. Starkregen, Wärmeinseln bei steigenden Temperaturen, verstärkte Hochwasserereignisse). Eine mögliche persönliche Betroffenheit wirkt sich dabei positiv auf die Bereitschaft aus, Maßnahmen umzusetzen.

Klimaanpassungshandeln ist zunächst individuell - man schützt sich und sein Hab und Gut. Die Entscheidung über die Art der geeigneten Maßnahmen und den Umgang damit wird durch die lokale und regionale Verwundbarkeit bedingt (z. B. Maßnahmen zum Hochwasserschutz). Die Einwohner Hamburgs fühlen sich sicher - auch vor den Auswirkungen des Klimawandels. Wa- rum also sein Verhalten ändern? Die neue Situation zu vermitteln, das Bewusstsein für die möglichen Risiken wachzuhalten und sich auf die Folgen des Klimawandels bereits zeitnah einzustellen, sich also anzupassen, wird eine öffentliche und private Aufgabe sein, der sich die Hamburger zu stellen haben.

Aus dem Stand der derzeitigen Forschung und den Ergebnissen der vorliegenden Studien lässt sich für die Zukunft ein Forschungsbedarf ableiten, der für das angepasste Risikomanagement, die Risikokommunikation und notwendige Klimaanpassungsmaßnahmen wichtig sein kann. So stellt sich z. B. für Hamburg die Frage, inwieweit es Unterschiede in der Klimawahrnehmung zwischen Bewohnern unterschiedlicher Stadtbezirke gibt bzw. ob sich unterschiedliche Sicherheitsgefühle in Bezug auf die Auswirkungen des Klimawandels feststellen lassen. Inwieweit ließe sich ein unterschiedliches Sicherheitsgefühl auf topographische Exponiertheit, auf technische Maßnahmen oder auf die mediale Präsentation zurückführen? Und welche Möglichkeiten sehen die Bürger, sich an die Folgen des Klimawandels anzupassen bzw. aus welchen Gründen tun sie dies (noch) nicht?

\section{Literatur}

Adger WN, Barnett J, Brown K, Marshal N, O'Brien K (2013) Cultural dimensions of climate change impacts and adaptation. Nat Clim Chang 3:112-117

Akerlof K, Maibach EW, Fitzgerald D, Cedeno AY, Neuman AEC (2012) Do people "personally experience" global warming, and if so how, and does it matter? Glob Environ Change 23:81-91

Arbuckle JG, Morton LW, Hobbs J (2013) Farmer beliefs and concerns about climate change and attitudes toward adaptation and mitigation: Evidence from lowa. Clim Change 118:551-563

Arlt D, Hoppe I, Wolling J (2010) Klimawandel und Mediennutzung. Wirkungen auf Problembewusstsein und Handlungsabsichten. Medien Kommunikationswiss 58(1):3-25

Balgar K, Mahlkow N (2013) Lokalkulturelle Konstruktionen von Vulnerabilität und Resilienz im Kontext des Klimawandels. IRS Working Paper 47.

Barnett J, Breakwell GM (2001) Risk perception and experience: hazard personality profiles and individual differences. Risk Anal 21(1):171-177

Birkholz S, Muro M, Jeffrey P, Smith HM (2014) Rethinking the relationship between flood risk perception and flood management. Sci Total Environ 478:12-20

Bock O, Schnapp K-U (2012) Politische Stimmung in der Freien und Hansestadt Hamburg: Ergebnisse einer allgemeinen Bevölkerungsumfrage. WiSo-HH Working Paper Series. Fakultät Wirtschafts- und Sozialwissenschaften $\mathrm{H}$ Lab Working Paper Nr 2. (Webseiten der Universität Hamburg)

Bostrom A, Morgan GM, Fischhoff B, Read D (1994) What do people know about global climate change? 1. mental models. Risk Anal 14(6):959-970

Brechin SR (2003) Comparative public opinion and knowledge on global climatic change and the Kyoto Protocol: the US versus the world? Int J Sociol Soc Pol 23:106-134

Brechin SR, Bhandari M (2011) Perceptions of climate change worldwide. WIREs Clim Chang 2(6):871-885

Brewer PR (2012) Polarisation in the USA: climate change, party politics, and public opinion in the Obama era. Eur Polit Sc 11(1):7-17

Brügger A, Dessai S, Devine-Wright P, Morton TA, Pidgeon NF (2015) Psychological responses to the proximity of climate change. Nat Clim Chang 5:1031-1037

Bürgerschaft der Freien und Hansestadt Hamburg (2013) Mitteilungen des Senats an die Bürgerschaft, Drucksache 20/8492 vom 25.6.2013

Capstick SB (2012) Climate change discourses in use by the UK public: commonalities and variations over a fifteen year period. Unpublished Doctoral Dissertation, Cardiff University, 2012. http://orca.cf.ac.uk/24182/. Zuletzt zugegriffen am 17.02.2016 
Capstick S, Whitmarsh L, Poortinga W, Pidgeon N, Upham P (2015) International trends in public perceptions of climate change over the past quarter century. WIREs Clim Chang 6(1):35-61

Chess C, Johnson BB (2007) Information is not enough. In: Moser SC, Dilling $\mathrm{L}$ (Hrsg) Creating a climate for change: Communicating climate change and facilitating social change. Cambridge University Press, Cambridge, $S$ 153-166

Christmann G, Heimann T, Mahlkow N, Balgar K (2012) Klimawandel als soziale Konstruktion? ZZF 1(1):20-36

Christmann G, Balgar K, Mahlkow N (2014) Local constructions of vulnerability and resilience in the context of climate change. A comparison of Lübeck and Rostock. Soc Sci 3(1):142-159

Crona B, Wutich A, Brewis A, Gartin M (2013) Perceptions of climate change: linking local and global perceptions through a cultural knowledge approach. Clim Change 119(2):519-531

Davenport C (2015) Climate Is Big Issue for Hispanics, and Personal. New York Times Feb 9, 2015 (Webseiten der New York Times)

Devine-Wright P (2013a) Think global, act local? The relevance of place attachments and place identities in a climate changed world. Glob Environ Chang 23:61-69

Devine-Wright $P$ (2013b) Dynamics of place attachment in a climate changed world. In: Manzo LC, Devine-Wright P (Hrsg) Place attachment. Routledge, New York, S 165-177

Devine-Wright P (2015a) Local attachments and identities: a theoretical and empirical project across disciplinary boundaries. Progr Hum Geogr 39(4):527-530

Devine-Wright P, Price J, Levionson Z (2015b) My country or my planet? Exploring the influence of multiple place attachments and ideological beliefs upon climate change attitudes and opinions. Glob Environ Chang 30:68-79

Dietz T, Stern PC (2008) Public participation in environmental assessment and decision making. National Academies Press, Washington, DC

Döring M, Ratter B (2017) The regional framing of climate change: Towards an 'emplaced' perspective on regional climate change perception in North Frisia. J Coast Conserv. Published online 28. Februar 2017. https://doi. org/10.1007/s11852-016-0478-0

Dunlap RE (1998) Lay perceptions of global risk: public views of global warming in cross-national context. Int Sociol 13:473-498

Engels A, Hüther O, Schäfer M, Held H (2013) Public climate-change skepticism, energy preferences and political participation. Glob Environ Chang 2(3):1018-1027

Grant S, Crim Tamason C, Mackie Jensen PK (2015) Climatization: a critical perspective of framing disasters as climate change events. Clim Risk Manag 10:27-34

Guber DL (2013) A cooling climate for change? Party polarization and the politics of global warming. Am Behav Sci 57:93-115

de Guttry C, Döring M, Ratter B (2016) Challenging the current climate change - migration nexus: exploring migrants' perceptions of climate change in the hosting country. Erde 147(2):109-118

Hartmuth G (2001) Soziale Repräsentationen des anthropogenen Klimawandels auf Sylt. Eine explorative Analyse lokal kontextualisierter Vorstellungen von Schlüsselpersonen. Dissertation, Universität Magdeburg, Fakultät für Geistes-, Sozial- und Erziehungswissenschaften

Heimann T, Christmann GB (2013) Klimawandel in den deutschen Küstenstädten und -gemeinden. Befunde und Handlungsempfehlungen für Praktiker. Leibniz Institute for Regional Development and Structural Planning, IRS Sonderpublikation, Erkner

Heimann T, Mahlkow N (2012) The social construction of climate adaptation governance: cultural differences in European coastal areas. Leibniz Institute for Regional Development and Structural Planning, Erkner

Heinrichs H, Grunenberg H (2007) Risikokultur - Kommunikation und Repräsentation von Risiken am Beispiel extremer Hochwasserrisiken. Endbericht für das BMBF. Leuphana Universität, Lüneburg

Heinrichs H, Grunenberg H (2009) Klimawandel und Gesellschaft. Perspektive Adaptionskommunikation. Springer VS, Wiesbaden

Heinrichs H, Grunenberg H, Knolle M (2007) Safecoast: the informed society. Final report. Schleswig-Holstein State Ministry for Agriculture, Environment and Rural Areas, Kiel (Webseiten der Europäischen Kommission, Projektseite Safecoast)
Hulme M (2009) Why we disagree about climate change: understanding controversy, inaction and opportunity. Cambridge University Press, Cambridge

Kahan DM, Peters E, Wittlin M, Slovic P, Ouellette LL, Braman D, Mandel G (2012) The polarizing impact of science literacy and numeracy on perceived climate change risks. Nat Clim Chang 2(10):732-735

Kaiser G, Reese S, Sterr H-J, Markau H (2004) COMRISK - common strategies to reduce the risk of storm floods in coastal lowlands - subprojekt 3: Public perception of coastal flood defence and participation in coastal flood defense planning - Final report. Department of Geography University of Kiel, Kiel

Kates RW, Wilbanks TJ (2003) Making the global local: responding to climate change concerns from the bottom up. Environment 45:12-23

Kempton W (1991) Lay perspectives on global climate change. Glob Environ Chang 1(3):183-208

Koerth J, Vafeidis AT, Carretero S, Sterr H, Hinkel J (2014) A typology of household-level adaptation to coastal flooding and its spatio-temporal patterns. Springerplus 3:466

Krapf H, Weller I (2013) Wahrnehmung des Klimawandels im Nordwesten. Ergebnisse einer repräsentativen Untersuchung zur Wahrnehmung von Klimawandel, Klimaschutz und Klimaanpassung in der Metropolregion Bremen-Oldenburg. In: Forschungszentrum Nachhaltigkeit (artec) (Hrsg) Artec-Paper Nr. 192. Forschungszentrum Nachhaltigkeit (artec), Bremen, S 1-87

Krosnick JA, Holbrook AL, Visser PS (2000) The impact of the fall 1997 debate about global warming on American public opinion. Public Underst Sci 9:239-260

Kruse N (2015) Klimawandel und Sturmfluten - Mentale Modelle von Expert*innen und Bürger*innen der Stadt Hamburg. In: Bundesamt für Bevölkerungsschutz und Katastrophenhilfe (Hrsg) Tagungsband LÜKEX 20153. Thementag: Herausforderungen großfächiger Evakuierungen, Bonn, $S$ $16-23$

Kruse N, Siedschlag D (2012) Sturmfluten und Küstenschutz an der schleswigholsteinischen Nordseeküste - eine vergleichende Betrachtung der Wahrnehmung von Bevölkerung und Experten. In: Bremer Beiträge zur Geographie und Raumplanung Beiträge der 29. Jahrestagung des Arbeitskreises "Geographie der Meere und Küsten. Bd. 44, S 120-129

Lavrillier A (2013) Climate change among nomadic and settled Tungus of Siberia: continuity and changes in economic and ritual relationships with the natural environment. Polar Rec 49:260-271

Leiserowitz AA (2004) Before and after - the day after tomorrow: a U.S. study of climate change risk perception. Environment 46(9):22-44

Leiserowitz AA (2005) American risk perceptions: Is climate change dangerous? Risk Anal 25:1433-1442

Leiserowitz AA (2007) Communicating the risks of global warming: American risk perceptions, affective images, and interpretive communities. In: Moser SC, Dilling L (Hrsg) Creating a climate for change: communicating climate change and facilitating social change. Cambridge University Press, Cambridge, S 44-63

Lorenzoni I, Pidgeon N (2006) Public views on climate change: European and USA perspectives. Clim Change 77(1-2):73-95

Lorenzoni I, Nicholson-Cole S, Whitmarsh L (2007) Barriers perceived to engaging with climate change among the UK public and their policy implications. Glob Environ Chang 17:445-459

Martens T, Garrelts H, Grunenberg H, Lange H (2009) Taking the heterogeneity of citizens into account: flood risk communication in coastal cities - a case study of Bremen. Nat Hazards Earth Syst Sci 9(6):1931-1940

Meusburger P (2006) Wissen und Raum - ein subtiles Beziehungsgeflecht. Bildung und Wissensgesellschaft. Heidelb Jahrb 49:269-308

Moser SC, Dilling L (2011) Communicating climate change: closing the science-action gap. In: Dryzek J, Norgaard R, Schlosberg D (Hrsg) The Oxford handbook of climate change and society. Oxford University Press, Cary, S 161-174

Myers TA, Maibach EW, Roser-Renouf C, Akerlof K, Leiserowitz AA (2012) The relationship between personal experience and belief in the reality of global warming. Nat Clim Chang 3(4):343-347

O'Neill SJ, Hulme M (2009) An iconic approach for representing climate change. Glob Environ Chang 19:402-410 
Paerregaard K (2013) Bare rocks and fallen angels: environmental change, climate perceptions and ritual practice in the Peruvian Andes. Religions (Basel) 4:290-305

Perkins S (2010) Atmospheric science: the cold facts. Nat Clim Chang. Published 09. November 2010. https://doi.org/10.1038/nclimate1008

Peters HP, Heinrichs H (2004) Interpretation des globalen Klimawandels durch die Öffentlichkeit. Konsequenzen für die Risikowahrnehmung und die Implementierung eines vorbeugenden Küstenschutzes; Projektbericht zum Teilprojekt 6 "Klimawandel und Öffentlichkeit" im Verbundvorhaben "Klimawandel und präventives Risiko- und Küstenschutzmanagement an der deutschen Nordseeküste" (KRIM) des deutschen Klimaforschungsprogramms DEKLIM, Schwerpunkt C "Klimawirkungsforschung". Technische Informationsbibliothek und Universitätsbibliothek Hannover, Forschungszentrum Jülich Programmgruppe MUT, Hannover, Jülich

Peters HP, Heinrichs H (2005) Öffentliche Kommunikation über Klimawandel und Sturmflutrisiken. Bedeutungskonstruktion durch Experten, Journalisten und Bürger. Schriften des Forschungszentrums Jülich, Reihe Umwelt, Bd. 58.

Plapp T, Werner U (2006) Understanding risk perception from natural hazards: examples from Germany. In: Amman WJ, Dannenmann ST, Vulliet L (Hrsg) Risk 21 - coping with risks due to natural hazards in the $21^{\text {st }}$ century. Taylor \& Francis, London, S 101-108

Raaijmakers R, Krywkow J, van der Veen A (2008) Flood risk perceptions and spatial multi-criteria analysis: an exploratory research for hazard mitigation. Nat Hazards 46(3):307-322

Ratter B, Kruse N (2010) Klimawandel und Wahrnehmung: Risiko und Risikobewusstsein in Hamburg. In: Böhner J, Ratter BMW (Hrsg) Klimawandel und Klimawirkung. Hamburger Symposium Geographie, Bd. 2. Institut für Geographie der Universität Hamburg, Hamburg, S 119-137

Ratter B, Sobiech C (2011) Heimat, Umwelt und Gefahren - Wahrnehmungen der Bevölkerung an der deutschen Nordseeküste. In: Fischer L, Reise K (Hrsg) Küstenmentalität und Klimawandel - Küstenwandel als kulturelle und soziale Herausforderung. oekom, München, S 181-196

Ratter B, Lange M, Sobiech C (2009) Heimat, Umwelt und Risiko an der deutschen Nordseeküste. Die Küstenregion aus Sicht der Bevölkerung. GKSSForschungszentrum, Geesthacht

Ratter B, Philipp K, von Storch H (2012) Between hype and decline: recent trends in public perception of climate change. Environ Sci Policy 18:3-8

Renn O (2008) Risk governance: coping with uncertainty in a complex world. Earthscan, London

Renn O, Rohrmann B (2000) Cross-cultural risk perception research: state and challenges. In: Renn O, Rohrmann B (Hrsg) Cross-cultural risk perception. A survey of empirical studies. Kluwer, Dordrecht, Boston, S 211-233

Renn O, Schweizer P, Dreyer M, Klinke A (2007) Risiko: Über den gesellschaftlichen Umgang mit Unsicherheit. oekom, München

Ryghaug M, Sørensen KH, Næss R (2011) Making sense of global warming: Norwegians appropriating knowledge of anthropogenic climate change. Public Underst Sci 20(6):778-795

Scannell L, Gifford R (2011) Personally relevant climate change: the role of place attachment and local versus global message framing in engagement. Environ Behav 45:60-85

Schmidt A, Striegnitz M, Kuhn K (2014) Integrating regional perceptions into climate change adaptation: a transdisciplinary case study from Germany's North Sea Coast. Reg Environ Chang 14(6):2105-2114

Shwom R, Bidwell D, Dan A, Dietz T (2010) Understanding US public support for domestic climate change policies. Glob Environ Chang 20:472-482

Sjöberg L (2000a) The methodology of risk perception research. Qual Quant 34(4):407-418

Sjöberg $L$ (2000b) Factors in risk perception. Risk Anal 20(1):1-11

Stehr N, von Storch H (2010) Klima, Wetter, Mensch. Barbara Budrich, Opladen

Sterman JD, Booth Sweeney L (2007) Understanding public complacency about climate change: Adults' mental models of climate change violate conservation of matter. Clim Chang 80(3-4):213-238

Taylor AL, Bruine de Bruin W, Dessai S (2014a) Climate change beliefs and perceptions of weather-related changes in the United Kingdom. Risk Anal 11(34):1995-2004
Taylor AL, Dessai S, Bruine de Bruin W (2014b) Public perception of climate risk and adaptation in the UK: a review of the literature. Clim Risk Manag 4-5:1-16

Trümper S, Neverla I (2013) Sustainable memory. How journalism keeps the attention for past disasters alive. Stud Commun Media 2(1):1-37

Upham P, Whitmarsh L, Poortinga W, Purdam K, Darnton A, McLachlan C, Devine-Wright $P$ (2009) Public attitudes to environmental change: a selective review of theory and practice. A research synthesis for the living with environmental change research programme. Research Councils UK, Swindon

Weber EU (2010) What shapes perceptions of climate change? WIREs Clim Chang 1:332-342

Weber M (2008) Alltagsbilder des Klimawandels: zum Klimabewusstsein in Deutschland. VS, Wiesbaden

Weber EU, Stern PC (2011) Public understanding of climate change in the United States. Am Psychol 66(4):315-328

Weller I, Krapf H, Wehlau D, Fischer K (2010) Untersuchung der Wahrnehmung des Klimawandels im Alltag und seiner Folgen für Konsumverhalten und Vulnerabilität in der Nordwest-Region. Eine explorative Studie. In: Forschungszentrum Nachhaltigkeit (artec) (Hrsg) Artec-Paper, artec-paper Nr. 166. artec, Bremen, S 1-103

Whitmarsh L (2008) Are flood victims more concerned about climate change than other people? The role of direct experience in risk perception and behavioural response. J Risk Res 11(3):351-374

Whitmarsh L (2011) Scepticism and uncertainty about climate change: dimensions, determinants and change over time. Glob Environ Chang 21:690-700

Wolf J, Moser SC (2011) Individual understandings, perceptions, and engagement with climate change: insights from in-depth studies across the world. WIREs Clim Chang 2:547-569

Open Access Dieses Kapitel wird unter der Creative Commons Namensnennung - Nicht kommerziell 4.0 International Lizenz (http://creativecommons.org/licenses/by-nc/4.0/deed.de) veröffentlicht, welche die nicht-kommerzielle Nutzung, Vervielfältigung, Bearbeitung, Verbreitung und Wiedergabe in jeglichem Medium und Format erlaubt, sofern Sie den/die ursprünglichen Autor(en) und die Quelle ordnungsgemäß nennen, einen Link zur Creative Commons Lizenz beifügen und angeben, ob Änderungen vorgenommen wurden.

Die in diesem Kapitel enthaltenen Bilder und sonstiges Drittmaterial unterliegen ebenfalls der genannten Creative Commons Lizenz, sofern sich aus der Abbildungslegende nichts anderes ergibt. Sofern das betreffende Material nicht unter der genannten Creative Commons Lizenz steht und die betreffende Handlung nicht nach gesetzlichen Vorschriften erlaubt ist, ist auch für die oben aufgeführten nicht-kommerziellen Weiterverwendungen des Materials die Einwilligung des jeweiligen Rechteinhabers einzuholen. 